\title{
Der Verlauf der endogenen Jahresrhythmik in Samen unter dem Einfluß verschiedenartiger Außenfaktoren
}

\author{
Von Erwin BünNing und Liselotte Müssle \\ Aus dem Botanischen Institut der Universität Tübingen \\ (Z. Naturforschg. 6 b, 108-112 [1951]; eingegangen am 20. Februar 1951)
}

\begin{abstract}
Die Geschwindigkeit des Ablaufs der endogenen Rhythmik, die in den Samen mancher Arten zu ungefähr jahresperiodischen Schwankungen der Keimbereitschaft führt, ist im Rahmen der erreichbaren Genauigkeit von der Temperatur unabhängig; selbst Lagerung der Samen bei $+45^{\circ}$ ergibt kein eindeutig früheres oder späteres Erreichen der Zeiten höchster und niedrigster Keimfähigkeit als Lagerung bei $-22^{\circ}$.

Auch Wasserentzug im Exsikkator, Lagerung in Sauerstoff, Stickstoff oder Kohlendioxyd, sowie starke Schädigung (vor dem Lagern) durch Hitze oder mechanische Einwirkungen beeinfiussen die Geschwindigkeit der Rhythmik nicht merklich.

Der Verlauf der Rhythmik ist deutlich abhängig von den Bedingungen, unter denen die Samen gereift sind. Ob dabei klimatische Einflüsse oder Einflüsse von der Mutterpflanze entscheidend sind, bleibt noch ungeklärt.
\end{abstract}

E ine vorhergehende Untersuchung ${ }^{1}$ hatte gezeigt, daß die endogene Jahresrhythmik in Samen eine Fortsetzung der endogenen Jahresrhythmik ist, die bei beblätterten Pflanzen am Wechsel von Ruhe- und Aktivitätsphasen beteiligt ist. Außerdem wurde gefunden, daß die Geschwindigkeit des Phasenwechsels der inneren Rhythmik in den Samen weitgehend von der Temperatur und vom Wassergehalt der Samen unabhängig ist. Dieser Versuch, durch Wahl unterschiedlicher Aufbewahrungsbedingungen und auch durch unterschiedliche Vorbehandlung den Ablauf der Rhythmik zu modifizieren und so einen Einblick in deren Natur zu gewinnen, wurde jetzt fortgesetzt.

Auch bei diesen neuen Versuchen wurde die Keimbereitschaft der unter konstanten Bedingungen gelagerten Samen wieder in Abständen von etwa einem Monat durch Auslegen von Proben (jeweils etwa 100 Samen) auf feuchtes Filtrierpapier in Petri-Schalen geprüft. Die Schalen standen im Dunkeln in einem auf $25^{\circ}$ geheizten Thermostaten.

\section{Einflußder Lagertemperatur}

Bei den früher veröffentlichten Versuchen konnte der Ablauf der Rhythmik bei Samen untersucht werden, die bei verschiedener konstanter Temperatur zwischen $+2^{\circ}$ und $+35^{\circ}$ gelagert waren. Da sich hierbei Unterschiede in der Geschwindigkeit der Periodizität

1 E. B ü nn in g, Z. Naturforschg. 4 b, 167 [1949]. Über jahresperiodische Keimfähigkeitsschwankungen vgl. außer den dort genannten Arbeiten noch: $\mathrm{H}$. E b n e r, Österr. Bot. Z. 73, 23 [1924], und W. S c h m i d t, Forsch. u. Fortschr. 25, 325 [1930].

nicht ergeben hatten, wurden jetzt noch stärker voneinander abweichende Lagertemperaturen geprüft. Die höchste Lagertemperatur betrug $+55^{\circ}$, die niedrigste $-22^{\circ}$.

a) Digitalis lutea, Ernte Mitte August 1949. Das erste Maximum der Keimfähigkeit (Ermittlung der nach 14 Tage langem Aufenthalt im Keimbett gekeimten Samen) und der Keimgeschwindigkeit (Ermittlung der schon nach 7 Tagen gekeimten) wird, wie Abb. 1-3 zeigen, Ende März 1950, d.h. 7 Monate nach der Ernte erreicht, einerlei, ob die Lagerung bei $-22^{\circ},+4^{\circ}$ oder $+45^{\circ}$ erfolgt. Die Kurven lassen eine Feststellung dieses Maximums natürlich nur mit einer Genauigkeit von etwa $\pm 1 / 2$ Monat zu. Das heißt, wir müssen die Möglichkeit offen lassen, daß das Maximum z. B. bei $+45^{\circ}$ etwa 1 Monat früher erreicht wird als bei einer Lagerung bei $-22^{\circ}$. Bei $+55^{\circ}$ (Abb. 4) wird das Maximum deutlich etwas früher erreicht. Aber der weitere Verlauf der Keimfähigkeitskurve zeigt, daß diese Temperatur schon schädigend wirkt: Die Keimfähigkeit geht allmählich verloren. Die Vorverlegung des Gipfels läßt also nicht mit Sicherheit eine Förderung im Ablauf der inneren Rhythmik erkennen, sondern beruht offenbar auf der Herabdrückung des späteren Keimprozents durch das Absterben in der zu hohen Lagertemperatur. Der zweite Gipfel hoher Keimfähigkeit wird zwischen Ende Oktober und Anfang Dezember erreicht, einerlei, ob die Lagerung bei $-22^{\circ},+4^{\circ}$ oder $+45^{\circ}$ erfolgt. Anscheinend bedingt die hohe Lagertemperatur eine geringe Verzögerung im Ablauf der inneren 

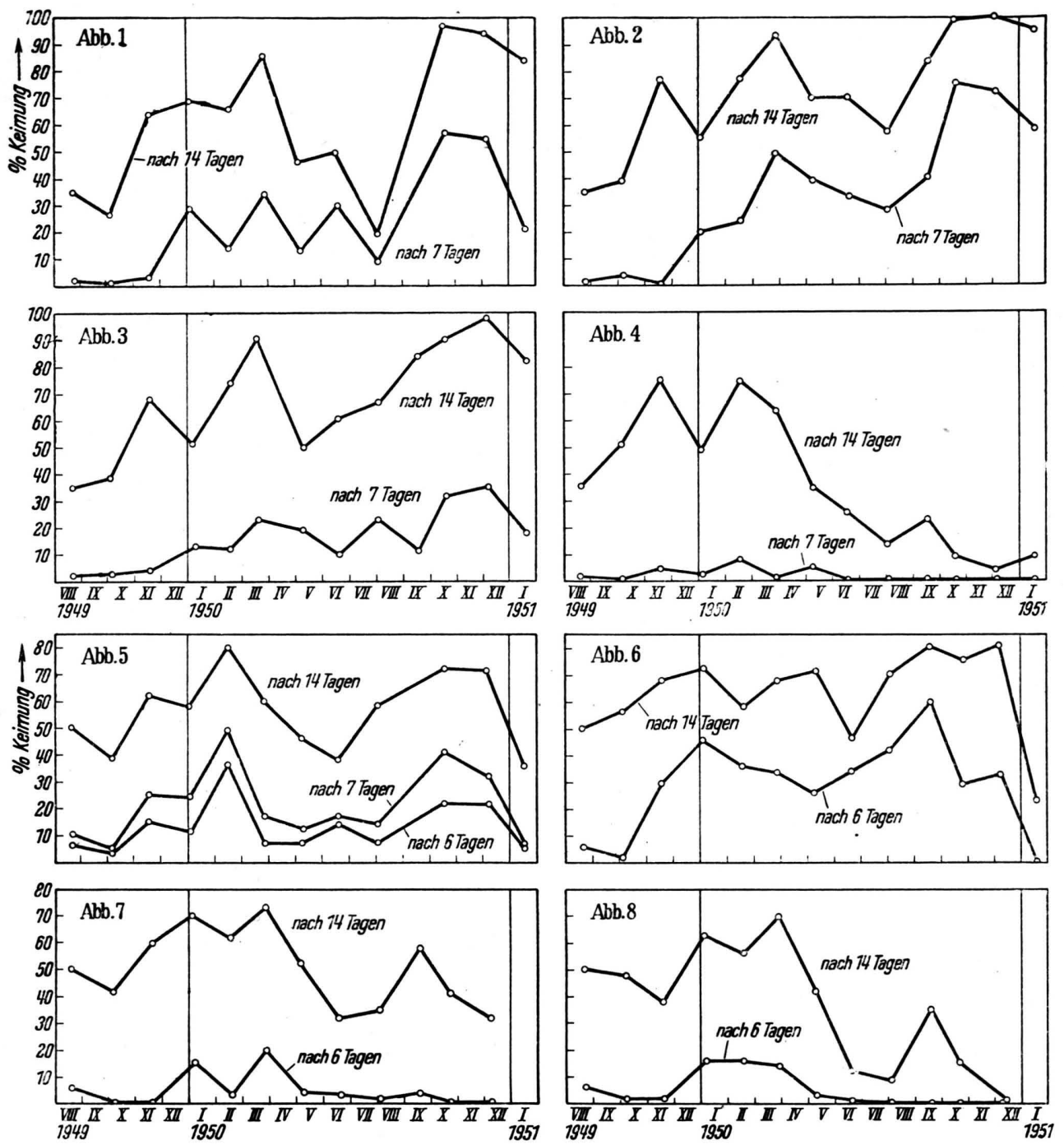

Abb. 1. Digitalis lutea. Lagerung bei $-22^{\circ}$. Angegeben ist der Prozentsatz der nach 7 und nach 14 Tage langem Aufenthalt im Keimbett erfolgten Keimungen. Abb. 2. Wie Abb. 1, aber Lagerung bei $+4^{\circ}$. Abb. 3. Wie Abb. 1, aber Lagerung bei $+45^{\circ}$. Abb. 4 . Wie Abb. 1, aber Lagerung bei $+55^{\circ}$. Abb. 5. Chrysanthemum corymbosum. Lagerung bei $-22^{\circ}$. Abb. 6. Wie Abb. 5, aber Lagerung bei $+4^{\circ}$. Abb. 7. Wie Abb. 5, aber Lagerung bei $+45^{\circ}$. Abb. 8. Wie Abb. 5, aber Lagerung bei $+55^{\circ}$.

Rhythmik (um vielleicht einen Monat, evtl. auch etwas mehr). Auch wenn dieser Unterschied reell ist, erweist er höchstens eine sehr geringe Temperaturabhängigkeit: Bei einem Temperaturunterschied von $67^{\circ}$ wurden im einen Fall 14-15, im anderen 15-16 Monate zur Erreichung der gleichen Phase der inneren Rhythmik benötigt. (Das bei $55^{\circ}$ gelagerte Material ist natürlich wegen der schon erwähnten Schädigung in diesem Zeitpunkt überhaupt nicht mehr auswertbar.)

Sehen wir jetzt noch auf das Minimum der Keimfähigkeit, das zwischen den beiden genannten Gipfeln liegt, so finden wir, daß dieser Zeitpunkt Anfang August erreicht wird, und zwar sowohl wenn die Lagertemperatur $-22^{\circ}$, als auch wenn sie $+4^{\circ}$ beträgt. Bei einer Lagertemperatur von $+45^{\circ}$ scheint das Minimum vorverlegt zu sein. Aber als sicher möchte ich diese Verschiebung nicht ansehen.

b) Chrysanthemum corymbosum. Das erste Maximum wird, wie Abb. 5 zeigt, ziemlich deutlich im Februar, das sind sechs Monate nach der Ernte, erreicht, wenn die Lagerung bei $-22^{\circ}$ erfolgte; Lagerung bei $+4^{\circ}$ kann das Maximum anscheinend um 
einen Monat vorverlegen (Abb. 6). Die bei 45 und $55^{\circ}$ gelagerten Samen (Abb. 7 u. 8) lassen das erste Maximum der Keimfähigkeit und Keimgeschwindigkeit nicht sehr deutlich erkennen, jedoch liegt es zwischen Februar und März. Das zweite Maximum wird etwa Ende Oktober bis Anfang November, d. h. wohl etwas mehr als 14 Monate nach der Ernte, erreicht, wenn die Lagerung bei $-22^{\circ}$ erfolgte. Lagerung bei $+4^{\circ}$ kann das Maximum anscheinend etwas vorverlegen. Bei 45 und $55^{\circ}$ ist diese Vorverlegung noch etwas ausgeprägter, aber aus den schon bei der Besprechung der Digitalis-Versuche erwähnten Gründen (allmähliches Absterben der Samen) ist diese Vorverlegung problematisch. Das Minimum zwischen den beiden Gipfeln wird immer ungefähr im Juni erreicht, einerlei, wie hoch die Lagertemperatur ist.

\section{Einflußdes Wasserentzugs}

Schon früher ${ }^{1}$ wurde versucht, durch einen Wasserentzug im Exsikkator den Verlauf der endogenen Rhythmik zu verändern. Jetzt wurde das noch einmal mit größerem Material, und zwar an Digitalis und Chrysanthemum überprüft. Wieder zeigte sich, daß eine deutliche Beeinflussung nicht möglich ist. Die Hoch- und Tiefpunkte der Keimfähigkeit wurden gleichzeitig mit denen der Kontrollen erreicht.

\section{Lagerung in verschiedenen Gasen}

Wenn am Zustandekommen der endogenen Rhythmik chemische Reaktionen beteiligt sind, könnte es möglich sein, die Geschwindigkeit des Verlaufs der Periodizität durch ständige Einwirkung eines anderen Gasmilieus, als es die atmosphärische Luft darstellt, zu modifizieren.

Für diese Versuche dienten Samen von Gratiola officinalis. Die für je eine Keimfähigkeitsprüfung bestimmten Proben (100 Samen) wurden in $5 \mathrm{~mm}$ weite, $15 \mathrm{~cm}$ lange Glasröhren verbracht und die Luft in den Röhren durch die betr. Gase ersetzt. Die Röhrchen wurden dann durch Zuschmelzen verschlossen und bei $+20^{\circ}$ im Dunkeln gelagert. In den üblichen Zeitabständen (etwa 1 Monat) wurde dann eines der Röhrchen entnommen und die Keimfähigkeit der in ihm enthaltenen Samen geprüft. In den Zeitabschnitten, in denen ein Anstieg der Keimfähigkeit zu erwarten war, wurde etwa alle 14 Tage eine Probe entnommen.

Die Kurven (Abb. 9-12) lassen den jahresperiodischen Verlauf der Keimfähigkeit erkennen. Das erste Maximum der Keimfähigkeit zeigt sich immer Anfang

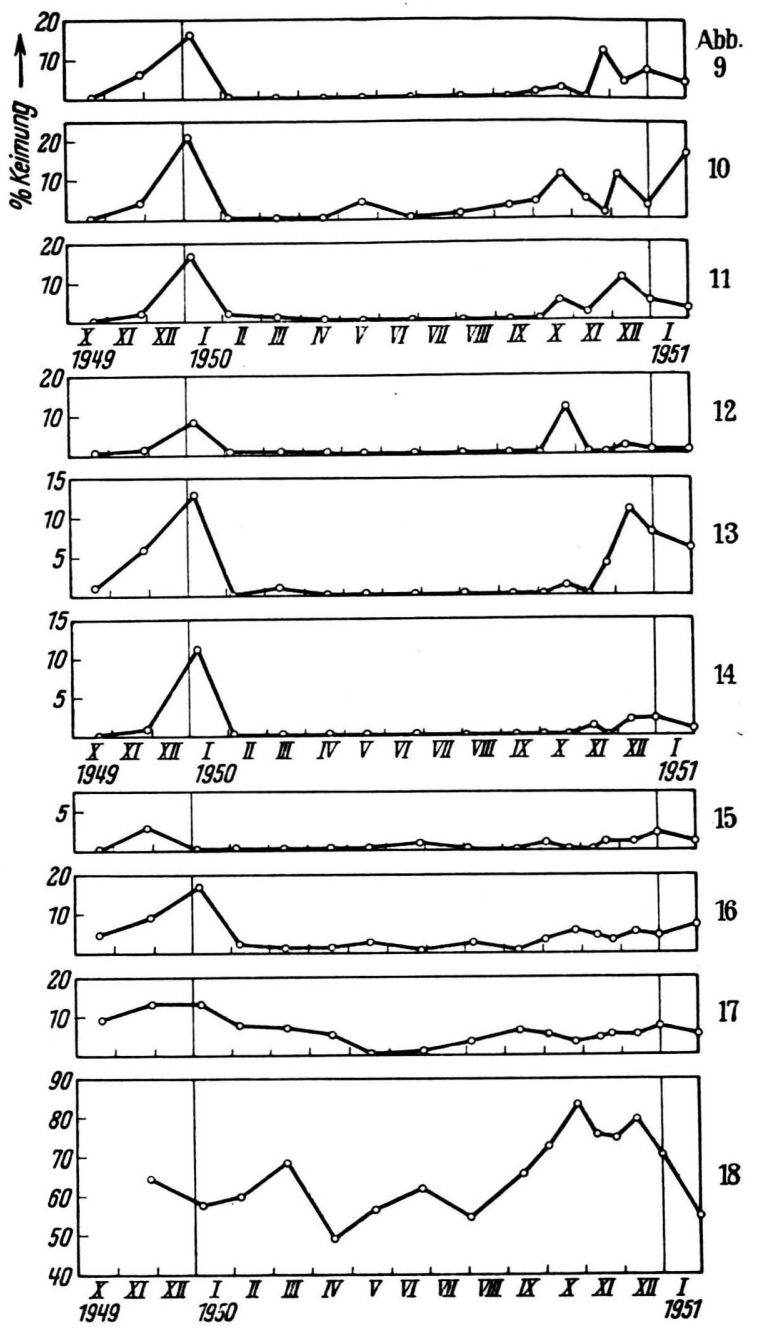

Abb. 9. Gratiola officinalis. Lagerung in Luft. Abb. 10. Wie Abb. 9, aber Lagerung in Sauerstoff. Abb. 11. Wie Abb. 9, aber Lagerung in Kohlendioxyd. Abb. 12. Wie Abb. 9, aber Lagerung in Stickstoff. Abb. 13. Gratiola officinalis. Vor der Lagerung 1 Min. auf $105^{\circ}$ erhitzt. Abb. 14. Wie Abb. 13, aber 10 Min. erhitzt. Abb. 15. Wie Abb. 13, aber 40 Min. erhitzt. Abb. 16. Gratiola officinalis. Samen vor der Lagerung mechanisch leicht beschädigt. Abb. 17. Wie Abb. 16, aber stärker beschädigt. Abb. 18. Gratiola officinalis. Samen vor jeder Keimprobe angestochen.

Januar, d. h. etwa $2^{1 / 2}$ Monate nach der Ernte. Das zweite Maximum war leider nicht so scharf ausgeprägt wie das erste, und nicht so scharf wie das zweite Maximum bei den früheren Versuchen mit Gratiola ${ }^{1}$. Jedoch zeigt sich bei allen Serien vom Oktober an, also etwa ein Jahr nach der Ernte, der Wiedereintritt der Keimfähigkeit. Nur bei den in Sauerstoff aufbewahrten Proben sind auch vorher schon einige Keimungen zu verzeichnen; eine Vorverlegung des Maximums scheint aber auch hier nicht erfolgt zu sein. 


\section{Hitzewirkung vor der Lagerung}

Bei Gratiola wurde auch noch systematischer als vorher ${ }^{1}$ bei Bellis perennis geprüft, ob eine Hitzewirkung auf die Samen, bevor die Lagerungszeit beginnt, den Ablauf der Rhythmik ändern kann. Die Samen wurden verschieden lange Zeit auf $105^{\circ}$ erhitzt, dann in Luft bei $+20^{\circ}$ im Dunkeln aufbewahrt. In allen Fällen lag sowohl das erste als auch das zweite Maximum der Keimfähigkeit ebenso wie bei den Kontrollen. Von den insgesamt durchgeführten 7 Serien (Erhitzung 1, 2, 5, 10, 20, 40 und 80 Min. lang) geben wir nur drei wieder (Erhitzung 1, 10 und 40 Min. lang). Alle Kurven (Abb. 13-15) zeigen, obwohl schon bei 10 Min. langer Erhitzung die Schädigung der Samen an der abnehmenden Keimfähigkeit deutlich wird, die Lage des ersten Maximums im Januar und das Wiedereintreten der Keimbereitschaft im Oktober oder November. (Die 80 Min. lang erhitzten Samen waren alle tot.)

\section{Wirkung mechanischer Beschädi-} gung

Da die Keimbereitschaft bei den Samen vieler Arten bekanntlich durch Entfernen, Anstechen oder sonstige Beschädigungen der Samenschale erhöht werden kann, wurde auch noch geprüft, ob sich die innere Rhythmik durch solche Behandlungen beeinflussen läßt. Die Samen wurden vor der Lagerung zwischen angerauhten Glasplatten gerieben und die Samenschale dadurch mehr oder weniger stark beschädigt. Diese Behandlung wurde gleich nach der Ernte (bei Gratiola officinalis) vorgenommen und das Material wie bei den vorher erwähnten Serien gelagert. Die Kurven (Abb. 15, 16) zeigen, daß sich die Keimbereitschaft durch diese Behandlung tatsächlich erhöhen läßt. Aber mindestens für das erste Maximum bleibt die zeitliche Lage unverändert, anscheinend auch für das zweite.

Ergänzend sei noch erwähnt, daß sich die Anzahl der Keimungen sehr stark erhöhen läßt, wenn die Samen (unmittelbar vor dem Ansetzen der Keimprobe) angestochen werden (Abb. 17). Die Rhythmik ist dann nicht mehr deutlich. Besonders überraschend ist dieses Ergebnis nicht. Wir können die Periodizität ja auch durch andere Mittel weitgehend verdecken, z. B. indem wir auf das Keimbett Licht einwirken lassen ${ }^{1}$. Daß mit solchen Einflüssen die Rhythmik nur verdeckt, nicht beseitigt ist, wurde außerdem noch durch die Beobachtung des Weiterwachsens der Keimlinge geprüft. Die nach mechanischer Beeinflussung oder durch Anstechen zu anomalen Zeiten erzwungenen Keimlinge zeigen nur ein sehr langsames Wachstum. Die innere Rhythmik hat ihren Sitz also zweifellos, wie auch schon früher begründet wurde, nicht in der Samenschale, sondern in den Embryonen. Besonders deutlich zeigte sich diese Lokalisierung der Rhythmik in den Embryonen übrigens bei Versuchen mit Fragaria vesca, die Frl. Mergenthaler in unserem Institut durchgeführt hat. Hier konnten die zu „anomalen Zeiten“ erzwungenen Keimlinge trotz sorgfältiger Pflege überhaupt nicht zur Weiterentwicklung gebracht werden; sie starben alle ab. Nur die in den Perioden hoher Keimbereitschaft gekeimten Pflanzen entwickelten sich weiter.
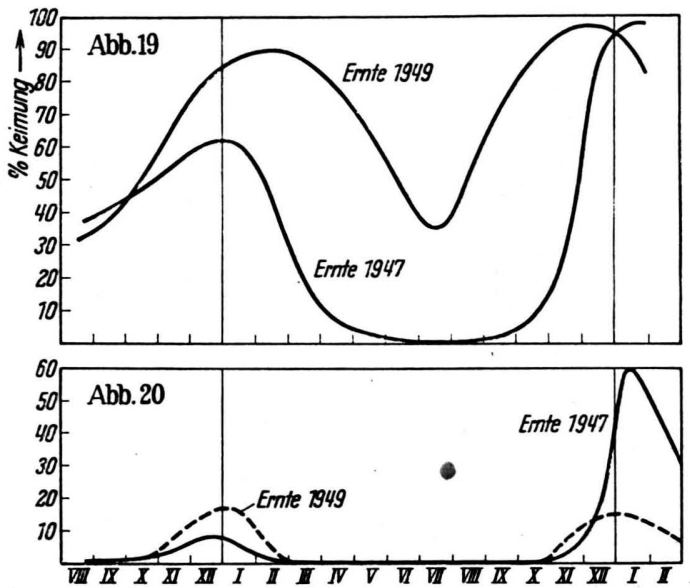

Abb. 19. Digitalis lutea. Verlauf der Keimbereitschaftsänderungen bei Samen der Ernte 1947 und 1949. Halbschematisch. Abb. 20. Wie Abb. 19, aber für Gratiola officinalis.

\section{Vergleich der $1947 / 49$ mit den $1949 / 51$ erzielten Ergebnissen}

Bei den Samen aller Arten, die wir in den vergangenen 5 Jahren untersucht haben, fiel uns auf, daß die Keimbereitschaftsschwankungen sich bei den Ernten der einzelnen Jahre mehr oder weniger voneinander unterscheiden. Auch für die in dieser Arbeit benutzten Arten trifft das zu. Wir begnügen uns damit, an Digitalis und Gratiola die Unterschiede im Verlauf der Rhythmik bei den 1947 und bei den 1949 geernteten Samen darzustellen (Abb. 19, 20).

Offenbar haben also die Bedingungen, unter denen die Samen an der Mutterpflanze gereift sind, einen Einfluß auf die spätere Periodizität der Keimbereitschaft. Wir können zunächst nicht entscheiden, ob dabei die Außenbedingungen wichtig sind, die während 
der Reifung herrschen, oder der innere Zustand der Mutterpflanzen. Beides erscheint uns möglich und einer experimentellen Prüfung wert.

\section{Diskussion}

Alle Versuche, die Geschwindigkeit der endogenen Rhythmik zu beeinflussen, sind somit mißlungen. Daher können bisher keine Vermutungen über die Natur der Prozesse, die in ihrer Wechselwirkung die Periodizität entstehen lassen, begründet werden.
Ein guter Ansatzpunkt für die weitere Analyse liegt aber in dem Befund, daß einige Besonderheiten im Verlauf der inneren Rhythmik von den Faktoren abhängen, die während der Samenreifung einwirkten.

Für die Unterstützung der Untersuchungen danken wir der Notgemeinschaft der Deutschen $\mathrm{W}$ is se $\mathrm{ns}$ ch a $\mathrm{t}$, für die Tiefkühllagerung des Materials Hrn. Dr. Wolff (Forschungsanstalt für Lebensmittelfrischhaltung in Karlsruhe), für fortgesetzte Hilfe beim Einsammeln des Materials und bei der Durchführung der Versuche Frl. Ruth Kautt.

\title{
Die Wirkung von Cumarin und Parasorbinsäure auf das Austreiben von Kartoffelknollen
}

\author{
Von Franí Moewus und Elisabeth Schader \\ Aus dem Max-Planck-Institut für medizinische Forschung, Heidelberg \\ (Z. Naturforschg. 6 b, 112--115 [1951]; eingegangen am 23. Februar 1951)
}

\begin{abstract}
Eine Keimungshemmung von Kartoffelknollen (Sorte „Ackersegen“) konnte erzielt werden: 1. durch Einwirkung von Cumarin-, Parasorbinsäure- und Sellerieöl-Dämpfen, 2. durch mehrmaliges, kurzfristiges Eintauchen der Knollen in 0,1-proz. wäßrige Parasorbinsäure- und Cumarinlösungen, 3. durch Bestreichen der Augen mit Wollfettpasten, die 0,1\% Cumarin enthielten. Die Zahl der austreibenden Augen bei dem Tauchverfahren konnte um 48\%, bei dem Pastenverfahren um 97\% gegenüber den Kontrollen reduziert werden. Bei den trotz der Behandlung ausgetriebenen Augen war sowohl das Sproßwachstum als auch die Bildung und das Wachstum der Wurzeln und Wurzelhaare deutlich verringert.
\end{abstract}

Ps arasorbinsäure, ein ungesättigtes Lacton, hemmt sowohl die Keimung von Kressesamen als auch das Wachstum von Kressewurzeln ${ }^{1}$. Auch Cumarin, das im Anschluß an die Untersuchung der Parasorbinsäure geprüft wurde, beeinflußt diese beiden Prozesse. Um festzustellen, welche entwicklungsphysiologisch wichtigen Vorgänge von Parasorbinsäure und Cumarin gehemmt werden können, wurden im Winter 1943/44 die Versuche auch auf Kartoffelknollen ausgedehnt ${ }^{2}$. Einerseits sollte festgestellt werden, ob diese Lactone überhaupt eine Wirkung entfalten, andererseits waren wir auf der Suche nach Objekten, an denen die hemmende Wirkung quantitativ erfaßt werden kann. Irgendein praktisches Ziel war mit diesen Versuchen nicht verbunden. Auch Det tweiler ${ }^{3}$ hat die Wirkung des Cumarins auf das Austreiben von Kartoffeln geprüft und betont, daß diese Beobachtungen nur theoretischen Wert haben. Für die

1 R. Kuhn, D. Jerchel, F. Moewus, E. F. Mölle r u. H. L e t tré, Naturwiss. 31, 468 [1943].

2 F. M o ew us, FIAT Review of German Science, Biochemie Teil II, 185 [1948].

3 Chr. Det tw e il e r, Chemische Methoden der Kartoffelkonservierung [1948].
Praxis kommen Präparate, die Wuchsstoffe enthalten (z. B. Belvitan K, Rhizopon C), oder solche, die Urethane enthalten (z. B. Agermin, Kikartol), in Betracht. Jedoch beobachtet man bei Verwendung derartiger Mittel immer wieder das Umschlagen der Hemmungswirkung in das Gegenteil ${ }^{4}$. Daß dies auch bei Cumarin und Parasorbinsäure möglich ist, haben Versuche über das Rhizoidwachstum von MarchantiaBrutkörpern ergeben ${ }^{5}$ : in genügend stark verdünnten Lösungen beider Lactone beobachtet man hier Wachstumsförderungen. Aus diesem Grunde sollten auch Präparate, die für die Praxis in Aussicht genommen sind, zuerst nach den von uns angewandten Methoden genauestens überprüft werden, damit später Fehlschläge vermieden werden. Grundsätzliche Angaben über die Wirkungsweise und den Nachweis von Keimungshemmstoffen finden sich a.a.O. ${ }^{6}$. Es ist bei solchen Versuchen auch der wechselnde Gehalt

${ }^{4}$ L. Quantz, Nachrichtenbl. biol. Zentralanst. Braunschweig 1, 8 [1949].

5 F. M o ew u s u. Elisabeth $\mathrm{S}$ ch ader, vgl. Beitr. z. Biol. d. Pfl. (im Druck).

6 F. M o ewus, Liselotte Moewus u. Elisabeth $\mathrm{S} \mathrm{ch}$ a d e r, vgl. diese Z. 6 b (im Druck). 